\title{
System Level Evaluation of Innovative Coded MIMO-OFDM Systems for Broadcasting Digital TV
}

\author{
Y. Nasser, J.-F. Hélard, and M. Crussière \\ Institute of Electronics and Telecommunications of Rennes, UMR CNRS, 6164, Rennes, France \\ Correspondence should be addressed to Y. Nasser, youssef.nasser@ieee.org
}

Received 28 March 2008; Revised 25 June 2008; Accepted 14 August 2008

Recommended by Marina Mondin

Single-frequency networks (SFNs) for broadcasting digital TV is a topic of theoretical and practical interest for future broadcasting systems. Although progress has been made in the characterization of its description, there are still considerable gaps in its deployment with MIMO technique. The contribution of this paper is multifold. First, we investigate the possibility of applying a space-time (ST) encoder between the antennas of two sites in SFN. Then, we introduce a 3D space-time-space block code for future terrestrial digital TV in SFN architecture. The proposed 3D code is based on a double-layer structure designed for intercell and intracell space time-coded transmissions. Eventually, we propose to adapt a technique called effective exponential signal-tonoise ratio (SNR) mapping (EESM) to predict the bit error rate (BER) at the output of the channel decoder in the MIMO systems. The EESM technique as well as the simulations results will be used to doubly check the efficiency of our 3D code. This efficiency is obtained for equal and unequal received powers whatever is the location of the receiver by adequately combining ST codes. The $3 \mathrm{D}$ code is then a very promising candidate for SFN architecture with MIMO transmission.

Copyright (c) 2008 Y. Nasser et al. This is an open access article distributed under the Creative Commons Attribution License, which permits unrestricted use, distribution, and reproduction in any medium, provided the original work is properly cited.

\section{INTRODUCTION}

Broadcasting digital TV is currently an area of intensive development and standardisation activities. The terrestrial broadcasting is the most challenging transmission system among the existing radio diffusion systems due to the presence of strong echoes.

Technically, single-frequency networks (SFNs) [1] present great advantages by transmitting lower power at various sites throughout the coverage area. In an SFN, the different antennas transmit the same signal at the same moment on the same carrier frequency. The existing SFN architectures are achieved in a single-input single-output (SISO) system since their deployment is very simple due to the use of one transmitting antenna by site. However, due to the increase of client services demand, it is desirable to deploy SFN with new MIMO techniques which ensure high spectrum efficiency as well as high diversity gain. The MIMO technique combined with the orthogonal frequency division multiplexing (OFDM) technique is pursued as a potential candidate for future generations of terrestrial portable and mobile digital video broadcasting related to DVB-T2 and
DVB-NGH proposals. Actually, one of the main research topics concerns the optimisation of the MIMO-OFDM schemes in order to obtain high-spectrum efficiency for high definition television (HDTV) services. In the literature, there are few studies on the SFN with MIMO transmission. The authors of [2] propose a new SFN model to increase the diversity gain in MIMO SFN architecture. In [3], an array antenna receiver using a maximum ratio combining technique is proposed to improve the system performance of the SFN transmission. This lack of studies on this original idea motivates our work to extend the application of the MIMO-OFDM transmission to the SFN architecture.

The optimisation of the MIMO-OFDM schemes in the SFN is highly desirable to be led in terms of the bit error rate (BER) after channel decoding. However, the optimisation of the MIMO-OFDM systems by simulations is time consuming. Thus, it is very important to accurately abstract the system level BER performance into analytical expression. Moreover, the system level performance abstraction should take into account the different transmission conditions, that is, modulation and coding scheme (MCS), synchronization errors, channel fading, and so forth. 
This paper presents a complete study on the optimisation of the MIMO-OFDM schemes for SFN architectures. The optimisation is double checked analytically and by simulations. This work has been carried out within the framework of a new European CELTIC project called Broadcast for 21st Century (B21C) project [4] and constitutes an extension of some previous works [5-7]. In this paper, we propose a 3D MIMO-OFDM scheme taking advantage of the particular characteristics of an SFN. More precisely, the contribution of this work is multifold. First of all, we investigate the possibility of applying a space-time block code (STBC) encoder between the antennas of two sites in SFN architecture. Secondly, using an iterative receiver, a generalized framework is proposed for modelling the effect of unbalanced powers received from different transmitting antennas in MIMOOFDM systems. This is a critical problem in SFN with mobile and portable reception. Another contribution of this work is the proposal of a new 3D space-time-space (STS) block code for SFN environment. The use of a second space dimension in the STS code will be justified as being particularly adapted and efficient in the case of SFN transmission. The proposed code is based on a double-level construction of ST coding resulting from the combination of two coding schemes: the intercell ST coding and the intracell ST coding. Eventually, we propose in this paper to adapt a technique, initially used for OFDM systems, to predict the BER at the output of the channel decoders of the MIMO-OFDM systems. This technique, called exponential effective SNR mapping (EESM) [7], is empiric but has been validated within the $3 \mathrm{GPP}$ project for the OFDM study item [8]. It provides a reliable link between link level simulations and system level simulations. In our contribution, we show that the EESM technique is independent of the MIMO scheme and of the power imbalance at the receiving side. It depends only on the modulation and coding scheme (MCS).

This paper is structured as follows. Section 3 describes the architecture of an SFN in which MIMO techniques are used. In Section 4, we present the transmission system model. Section 5 presents the receiving model with iterative receiver. In Section 6, we discuss the construction of different STBC schemes considered in this paper and we describe our proposed 3D code for MIMO-OFDM transmission. In Section 7, we adapt an accurate abstraction of the system level BER performance, initially proposed for the OFDM systems, to the MIMO-OFDM systems. In Section 8, we double check the efficiency of the proposed 3D code using the BER abstraction method described in Section 7. Conclusions are drawn in Section 8.

\section{MIMO SYSTEMS IN SFN}

In this paper, we propose to apply a MIMO communication scheme between the antennas located in the different sites of an SFN architecture. Such a system could be implemented using $M_{T}$ transmit antennas ( $\mathrm{Tx}$ ) by site as shown in Figure 1. Without loss of generality, we will consider in our study the transmission behaviour of two neighbouring cells using a total of $\left(2 \times M_{T}\right)$ Tx and $M_{R}$ receive antennas (Rx).

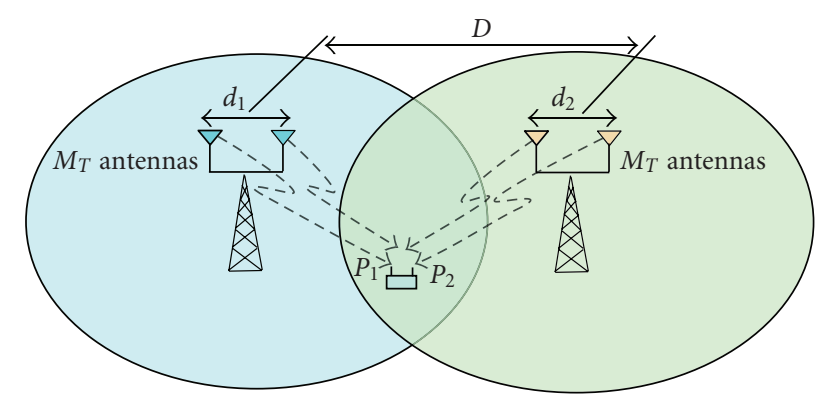

FigURE 1: SFN with unequal received powers.

The extension of our study to more sites could be adequately adapted.

Classically, in SFN architectures, the different antennas transmit at the same moment the same signal on the same frequency. For the SFN to work properly, the resulting delay spread $\tau_{\max }$ of the different received signals must be less than the duration of the guard interval (GI) time inserted at the beginning of each OFDM symbol.

As a starting point, let us assume that each site holds one antenna and that the receiver receives signals from both antennas. In the case of an SFN, the time offset between the signals received from each site antennas could be seen as a superposition of the time offset between transmitters' signals (the signal time delay between the transmitting antennas) and the signal time offset between each transmitter and the receiver. The first offset is generally negligible since the transmitters are synchronized with an ultrastable reference like the global positioning system (GPS). The second offset could be seen as follows. When the mobile terminal (MT) moves within one cell, it receives signal from its own cell antenna but also from the neighbouring cell antenna. Since the MT is not equidistant to both antennas, the signal received from each one will be delayed according to the position of the MT. This results into a delay $\Delta \tau$ between the two signals received from both antennas or equivalently between the channel impulse responses (CIRs) between the transmitters and the receiver. The delays are directly related to the distances between the transmitters and the receiver and thus to the signal strength ratio at the receiver. Assuming an equal transmitted power $P_{0}$ at each antenna, the received power from the $i$ th antenna is

$$
P_{i}=\frac{P_{0}}{d_{i}^{\alpha}}
$$

where $d_{i}$ is the distance between the receiver and the $i$ th transmitter and $\alpha$ is the propagation constant which depends on the transmission environment.

The delay of each CIR between the ith transmitter and the receiver is

$$
\tau_{i}=\frac{d_{i}}{c}
$$

where $c$ is the light velocity.

Without loss of generality, let us assume that the first transmitter site is the reference site. Substituting $d_{i}$ from (2) 


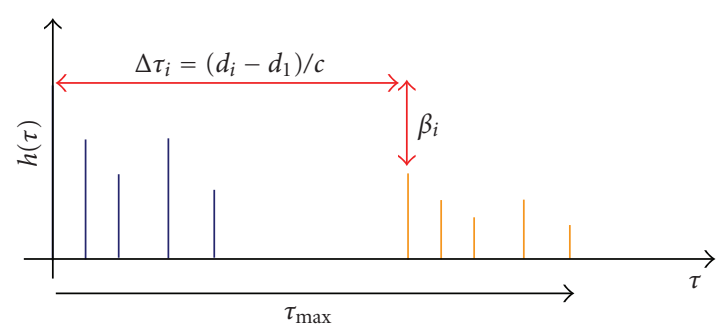

Figure 2: SFN with unequal received powers.

in (1), the CIR delay of the $i$ th link (i.e., between the $i$ th transmitter and the receiver) with respect to the reference antenna can be expressed by

$$
\Delta \tau_{i}=\tau_{i}-\tau_{1}=\left(10^{-\beta_{i} / 10 \alpha}-1\right) \frac{d_{1}}{c},
$$

where $d_{1}$ is the distance between the reference transmitter (first one) and the receiver; and $\beta_{i}$ is the received power difference (expressed in $\mathrm{dB}$ ) between the signal received from the reference site and the signal received from the $i$ th transmitter. It is given by

$$
\beta_{i}[\mathrm{~dB}]=-10 \cdot \alpha \cdot \log _{10}\left(\frac{d_{i}}{d_{1}}\right) .
$$

In the sequel, we will assume that the power received from the reference antenna is equal to $0 \mathrm{~dB}$ and the distance $d_{i}$ is greater than $d_{1}$ whatever $i>1$. It is a real situation where the MT is closer to its own cell antenna than to the other antennas. In this case, $\beta_{i}$ is neither than the power attenuation factor between the $i$ th transmitter and the MT. As a consequence, the transmission model becomes equivalent to a system with unbalanced powers received from each site antennas. Figure 2 shows an example of the relation between the power attenuation factor $\beta$ and the CIR delay of the $i$ th link with respect to the reference antenna signal.

If we now consider that the number of $\mathrm{Tx}$ in one site is greater than one (i.e., $M_{T}>1$ ), the choice of an adequate MIMO scheme should then be based on this imbalance. Moreover, it should be adequate for intercell environment, (i.e., between antennas signals of each site) and intracell environment (i.e., between antennas signals in each site). Furthermore, it should be chosen adequately to cope with equal and unequal received powers. This will be the subject of Section 6 where we propose a 3D STS code adapted to such situations.

We note that in this paper we consider independent CIRs with the dominant problem of the SFN architecture, that is, the problem of the CIR delays and the power loss. However, in real situations, other problems like CIRs correlations should be considered also. The reader may refer to [9] and the references therein for more details.

\section{TRANSMISSION MODEL}

In this section, we describe the transmission model of the double-layer STBC constructed between the antennas of the different sites. The double layer proposed here has to cope with the equal and unequal received powers. The first layer in our proposed code corresponds to the intercell ST coding while the second corresponds to the intracell ST coding.

Figure 3 depicts the transmitter modules at each site. Information bits $b_{k}$ are first channel encoded, randomly interleaved, and fed to a quadrature amplitude modulation (QAM) module. We recall that we restrict our study to two sites only and the generalisation could be done in different forms. Therefore, the SFN transmission system involving the two sites (described in Figure 1) could be seen as a double-layer scheme in the space domain. The first layer is seen between the 2 sites separated by $D \mathrm{~km}$. The second layer is seen between the antennas separated by $d$ meters within one site. For the first layer, a space time block code (STBC) scheme is applied between the two signals transmitted by each site antennas. In the second layer, we use a second STBC encoder for each subset of $M_{T}$ signals transmitted from the same site. For the first layer (resp., the second layer), the STBC encoder takes $L$ (resp., $M$ ) sets of data complex symbols and transforms them into a $(2, U)$ (resp., $\left.\left(M_{T}, V\right)\right)$ output matrix according to the STBC scheme. This output is then fed to $2 \times M_{T}$ OFDM modulators, each using $N_{c}$ subcarriers. In order to have a fair analysis and comparison between different STBC codes, the signal power at the output of the ST encoder is normalized by $2 \times M_{T}$.

The double-layer encoding matrix of the proposed code is described by

$$
\begin{aligned}
\mathbf{X}^{(1)} & =\left(\begin{array}{ccc}
\mathbf{X}_{11}^{(2)} & \cdots & \mathbf{X}_{1 U}^{(2)} \\
\mathbf{X}_{21}^{(2)} & \ldots & \mathbf{X}_{2 U}^{(2)}
\end{array}\right) \\
\mathbf{X}_{p q}^{(2)} & =\left(\begin{array}{ccc}
f_{p q, 11}\left(s_{1}, \ldots s_{M}\right) & \ldots & f_{p q, 1 V}\left(s_{1}, \ldots s_{M}\right) \\
\vdots & \ddots & \vdots \\
f_{p q, M_{T} 1}\left(s_{1}, \ldots s_{M}\right) & \cdots & f_{p q, M_{T} V}\left(s_{1}, \ldots s_{M}\right)
\end{array}\right) .
\end{aligned}
$$

In (5), the superscript indicates the layer, $f_{p q, i t}\left(s_{1}, \ldots s_{M}\right)$ is a function of the input complex symbols $s_{m}$ and depends on the STBC encoder scheme. The subscripts $p$ and $q$ are such that $p=1,2$ and $q=1, \ldots, U$. They reflect, respectively, the STBC encoder input size at each layer. The time dimension of the resulting $3 \mathrm{D}$ code is equal to $U \times V$ and the resulting coding rate is $R=(L \times M) /(U \times V)$.

In order to simplify the transmission model, the doublelayer encoding matrix given in (5) will be represented by $\mathbf{X}=\left[x_{i, t}\right]$, where $x_{i, t}\left(i=1, \ldots, 2 \times M_{T} ; t=1, \ldots, U \times\right.$ $V)$ is the output of the double-layer STBC encoder on a given subcarrier $n$. In other words, the layers construction is transparent from the transmission model viewpoint. Moreover, we set $Q=L \times M$ vas the number of the complex symbols at the input of the double-layer STBC encoder and we set $T=U \times V$ as the number of the corresponding output symbols. The ST coding rate is then $R=Q / T$. 


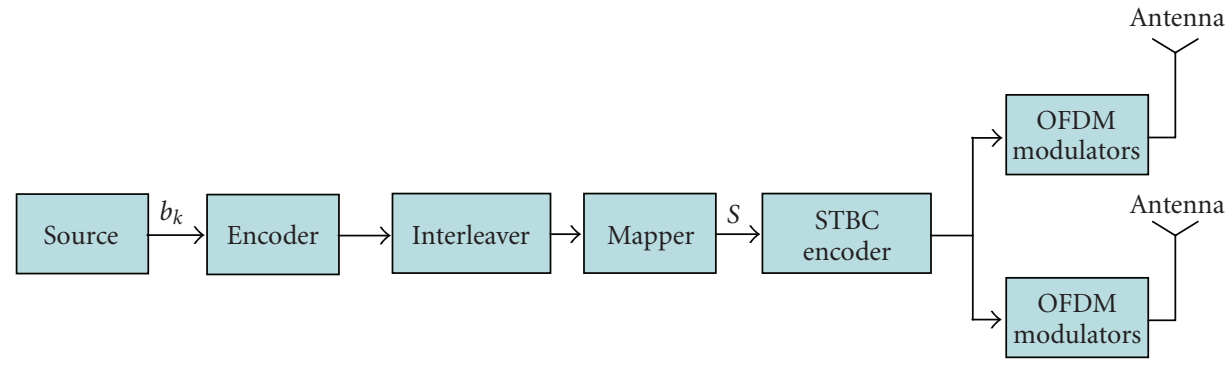

FIgURE 3: MIMO-OFDM transmitter.

\section{ITERATIVE STBC RECEIVER}

\subsection{Receiving model}

We assume that the transmitter and the receiver are perfectly synchronised. Moreover, we assume perfect channel state information (CSI) at the receiver. In this paper, the transmission is described in frequency domain for simplicity reasons. However, in real scenario, the signal is transferred to the time domain and cyclic prefix (CP) insertion operations are achieved at the transmitting side. Reciprocal operations are done at the receiving side. The signal received on the subcarrier $n$ by the antenna $j$ is a superposition of the transmitted signal by the different antennas multiplied by the channel coefficients $h_{j, i}[n]$ (" $i$ " is the index of the transmitting antenna) to which additive white Gaussian noise (AWGN) is added. It is given by

$$
y_{j, t}[n]=\sum_{i=1}^{2 M_{T}} \sqrt{P_{i}} h_{j, i}[n] x_{i, t}[n]+w_{j, t}[n],
$$

where $y_{j, t}[n]$ is the signal received on the $n$th subcarrier by the $j$ th receiving antenna during the $t$ th OFDM symbol period. $h_{j, i}[n]$ is the frequency channel coefficient assumed to be constant during $T$ symbol durations, $x_{i, t}[n]$ is the signal transmitted by the ith antenna, and $w_{j, t}[n]$ is the additive AWGN with zero mean and variance $\mathrm{N}_{0} / 2$. In the sequel, we will drop the subcarrier index $n$ for simplicity. By introducing an equivalent receive matrix $\mathbf{Y} \in \mathbb{C}^{M_{R} \times T}$ whose elements are the complex received symbols expressed in (6), we can write the received signal on the $n$th subcarrier on all receiving antennas as

$$
\mathbf{Y}=\mathbf{H P X}+\mathbf{W}
$$

where $\mathbf{H}$ is the $\left(M_{R}, 2 M_{T}\right)$ channel matrix whose components are the coefficients $h_{j, i}, \mathbf{P}$ is a $\left(2 M_{T}, 2 M_{T}\right)$ diagonal matrix containing the signal magnitudes $\sqrt{P_{i}}, \mathbf{X}$ is a $\left(2 M_{T}, T\right)$ complex matrix containing the transmitted symbols $x_{i}[t] . \mathrm{W}$ is a $\left(M_{R},, T\right)$ complex matrix corresponding to the AWGN.

Let us now describe the transmission link with a general model independently of the ST coding scheme. We separate the real and imaginary parts of the complex symbols input vector $\mathbf{s}\left\{s_{q}: q=1, \ldots, Q\right\}$ of the outputs $\mathbf{X}$ of the doublelayer ST encoder as well as those of the channel matrix $\mathbf{H}$, and the received signal $\mathbf{Y}$. Let $s_{q, \mathcal{R}}$ and $s_{q, \mathcal{g}}$ be the real and imaginary parts of $s_{q}$. The main parameters of the double code are given by its dispersion matrices $\mathbf{U}_{\mathbf{q}}$ and $\mathbf{V}_{\mathbf{q}}$ corresponding (not equal) to the real and imaginary parts of $\mathbf{X}$, respectively. With these notations, $\mathbf{X}$ is given by

$$
\mathbf{X}=\sum_{q=1}^{Q}\left(s_{q, \Re} \mathbf{U}_{\mathbf{q}}+j s_{q, \mathfrak{I}} \mathbf{V}_{\mathbf{q}}\right),
$$

where $\mathbf{U}_{\mathbf{q}}$ is the dispersion matrix having the same dimensions of $\mathbf{X}$ such that

$$
\mathbf{U}_{\mathbf{q}}(m, n)= \begin{cases}1, & \text { if } \mathbf{X}(m, n)=s_{q, \Re}, \\ -1, & \text { if } \mathbf{X}(m, n)=-s_{q, \Re}, \\ 0, & \text { elsewhere, }\end{cases}
$$

where $\mathbf{V}_{\mathbf{q}}$ could be deduced from (9) by replacing the real part by the imaginary part.

In the sequel, we separate the real and imaginary parts of $\mathbf{S}, \mathbf{Y}$, and $\mathbf{X}$, and stack them row-wise in vectors of dimensions $(2 Q, 1),\left(2 M_{R} T, 1\right)$, and $\left(4 M_{T} T, 1\right)$, respectively. We obtain

$$
\begin{gathered}
\mathbf{s}=\left[s_{1, \mathfrak{R}}, s_{1, \mathfrak{I}}, \ldots, s_{Q, \Re}, s_{Q, \mathfrak{I}}\right]^{\mathrm{T}}, \\
y=\left[y_{1, \Re}, y_{1, \mathfrak{I}}, \ldots, y_{T, \mathfrak{R}}, y_{T, \mathfrak{I}}, \ldots, y_{M_{R} T, \Re}, y_{M_{R} T, \mathfrak{I}}\right]^{\mathrm{T}}, \\
\mathbf{x}=\left[x_{(1,1), \Re}, x_{(1,1), \mathfrak{I}}, \ldots, x_{\left(2 M_{T}, T\right), \Re}, x_{\left(2 M_{T}, T\right), \mathfrak{I}}\right]^{\mathrm{T}},
\end{gathered}
$$

where $[\cdot]^{\mathrm{T}}$ holds for matrix transpose.

Since we use linear ST coding, the vector $\mathbf{x}$ can be written as

$$
\mathbf{x}=\mathbf{F} \cdot \mathbf{s},
$$

where $\mathbf{F}$ has the dimensions $\left(4 M_{T} T, 2 Q\right)$ and is obtained through the dispersion matrices of the real and imaginary parts of s. It is given by

$$
\mathbf{F}=\left[\begin{array}{cccc}
\mathbf{F}_{1}(1,1) & \cdots & \cdots & \mathbf{F}_{\mathbf{Q}}(1,1) \\
\vdots & \vdots & \vdots & \vdots \\
\mathbf{F}_{1}(1, T) & \cdots & \cdots & \mathbf{F}_{\mathbf{Q}}(1, T) \\
\vdots & \ddots & \vdots & \vdots \\
\mathbf{F}_{1}\left(2 M_{T}, T\right) & \cdots & \cdots & \mathbf{F}_{\mathbf{Q}}\left(2 M_{T}, T\right)
\end{array}\right] \text {, }
$$


where $\mathbf{F}$ is composed of $2 M_{T}$ blocks of $2 T$ rows each, that is, the data transmitted on each antenna is gathered in one block having $2 T$ rows and $2 Q$ columns according to the ST coding scheme. The different components of $\mathbf{F}$ are given by

$$
\mathbf{F}_{\mathbf{q}}(m, t)=\left[\begin{array}{cc}
\mathbf{U}_{\mathbf{q}, \Re}(m, t) & -\mathbf{V}_{\mathbf{q}, \mathfrak{I}}(m, t) \\
\mathbf{U}_{\mathbf{q}, \mathfrak{I}}(m, t) & \mathbf{V}_{\mathbf{q}, \Re}(m, t)
\end{array}\right] .
$$

As we change the formulation of $\mathbf{S}, \mathbf{Y}$, and $\mathbf{X}$ in (10), it can be shown that vectors $\mathbf{x}$ and $\mathbf{y}$ are related through the matrix
G of dimensions $\left(2 M_{R} T, 4 M_{T} T\right)$ such that

$$
\mathbf{y}=\mathbf{G B x}+\mathbf{w}
$$

The matrix $\mathbf{B}$ is a $\left(4 M_{T} T, 4 M_{T} T\right)$ diagonal matrix whose components are given by

$$
\begin{gathered}
B_{i, i}=\sqrt{P_{i}} \quad 2 \cdot T(p-1)+1 \leq i \leq 2 T \cdot p, \\
p=1, \ldots, 2 M_{T} .
\end{gathered}
$$

Matrix $\mathbf{G}$ is composed of blocks $\mathbf{G}_{\mathbf{j}, \mathbf{i}}\left(j=1, \ldots, M_{R} ; i=\right.$ $\left.1, \ldots, 2 M_{T}\right)$ each having $(2 T, 2 T)$ elements given by

$$
\mathbf{G}_{\mathbf{j}, \mathbf{i}}=\left(\begin{array}{cccc}
h_{(j, i), \Re} & -h_{(j, i), \mathfrak{I}} & 0 & \\
h_{(j, i), \mathfrak{I}} & h_{(j, i), \Re} & 0 & \\
0 & 0 & h_{(j, i), \Re} & -h_{(j, i), \mathfrak{I}} \\
0 & 0 & h_{(j, i), \mathfrak{I}} & h_{(j, i), \Re} \\
0 & & \ldots & 0 \\
0 & & \ldots & 0 \\
0 & & \ldots & \\
0 & & \ldots &
\end{array}\right.
$$

$$
\left.\begin{array}{cccc} 
& \cdots & & 0 \\
& \cdots & & 0 \\
0 & \cdots & & 0 \\
0 & \cdots & & 0 \\
\ddots & & 0 & 0 \\
& \ddots & 0 & 0 \\
& 0 & h_{(j, i), \Re} & -h_{(j, i), \mathfrak{I}} \\
& 0 & h_{(j, i), \mathfrak{I}} & h_{(j, i), \Re}
\end{array}\right)_{(2 T, 2 T)}
$$

Now, substituting $\mathbf{x}$ from (11) in (14), the relation between $\mathbf{y}$ and $s$ becomes

$$
\mathrm{y}=\mathrm{GB} F \mathbf{s}+\mathbf{w}=\mathrm{G}_{\mathrm{eq}} \mathbf{s}+\mathbf{w}
$$

where $\mathbf{G}_{\mathbf{e q}}$ is the equivalent channel matrix between $\mathbf{s}$ and $\mathbf{y}$. It is assumed to be known perfectly at the receiving side.

\subsection{STBC detector}

The detection problem is to find the transmitted data $\mathbf{s}$ given the vector $y$. In the case of orthogonal STBC (OSTBC), the optimal receiver is made of a concatenation of ST decoder and channel decoder modules. In nonorthogonal STBC (NO-STBC) schemes, there is an interelement interference (IEI) at the receiving side. The optimal receiver in this case is based on joint ST and channel-decoding operations. However, such receiver is extremely complex to implement and requires large memory to store the different points of the trellis. Moreover, it could not be implemented reasonably in one chip. Thus, the suboptimal solution proposed here consists of an iterative receiver where the ST detector and channel decoder exchange extrinsic information in an iterative way until the algorithm converges. The iterative detector shown in Figure 4 is composed of a parallel interference canceller (PIC), a demapper which consists in computing the soft information of the transmitted bits, that is, a log likelihood ratio (LLR) computation [10], a soft-input softoutput (SISO) decoder [11], and a soft mapper.

At the first iteration, the demapper takes the estimated symbols $\widehat{\mathbf{s}}$, the knowledge of the channel $\mathbf{G}_{\mathbf{e q}}$, and of the noise variance, and computes the LLR values of each of the coded bits transmitted per channel use. The estimated symbols $\widehat{\mathbf{s}}$ are obtained via minimum mean square error (MMSE) filtering according to

$$
\hat{s}_{p}^{(1)}=\mathbf{g}_{\mathbf{p}}^{\mathbf{t r}}\left(\mathbf{G}_{\mathrm{eq}} \cdot \mathbf{G}_{\mathbf{e q}}^{\mathbf{t r}}+\sigma_{w}^{2} \mathbf{I}\right)^{-1} \mathbf{y},
$$

where $\mathbf{g}_{\mathbf{p}}^{\mathrm{tr}}$ of dimension $\left(2 M_{R} T, 1\right)$ is the $p$ th column of $\mathbf{G}_{\text {eq }}(1 \leq p \leq 2 Q) . \hat{s}_{p}^{(1)}$ is the estimation of the real part ( $p$ odd) or imaginary part ( $p$ even) of $s_{q}(1 \leq q \leq Q)$. Once the estimation of the different symbols $s_{q}$ is achieved by the soft mapper at the first iteration, we use this estimation for the next iterations process.

From the second iteration, we perform PIC operation followed by a simple inverse filtering (instead of MMSE filtering at the first iteration):

$$
\hat{\mathbf{y}}_{\mathbf{p}}=\mathbf{y}-\mathbf{G}_{\mathbf{e q}, \mathbf{p}} \widetilde{\mathbf{s}}_{\mathbf{p}}^{(1)}, \quad \hat{\boldsymbol{s}}_{p}^{(2)}=\frac{1}{\mathbf{g}_{\mathbf{p}}^{\operatorname{tr}} \mathbf{g}_{\mathbf{p}}} \mathbf{g}_{\mathbf{p}}^{\operatorname{tr}} \hat{\mathbf{y}}_{\mathbf{p}},
$$

where $\mathbf{G}_{\mathbf{e q}, \mathbf{p}}$ of dimension $\left(2 M_{R} T, 2 Q-1\right)$ is the matrix $\mathbf{G}_{\mathbf{e q}}$ with its $p$ th column removed, $\widetilde{s}_{\mathbf{p}}(\mathbf{1})$ of dimension $(2 Q-1,1)$ is the vector $\tilde{\mathbf{s}}$ estimated by the soft mapper with its $p$ th entry removed.

\section{3D STSBC CONSTRUCTION}

The aim of this section is to judiciously build the proposed double-layer 3D STS code so that the resulting MIMO scheme behaves efficiently in an SFN context. We then need to choose the adequate ST coding scheme to apply to each layer of our 3D code. In the sequel, we will consider different coding schemes to apply to the different layers. First, we will consider the well-known orthogonal Alamouti ST coding scheme [12] for its robustness and its simplicity. In 


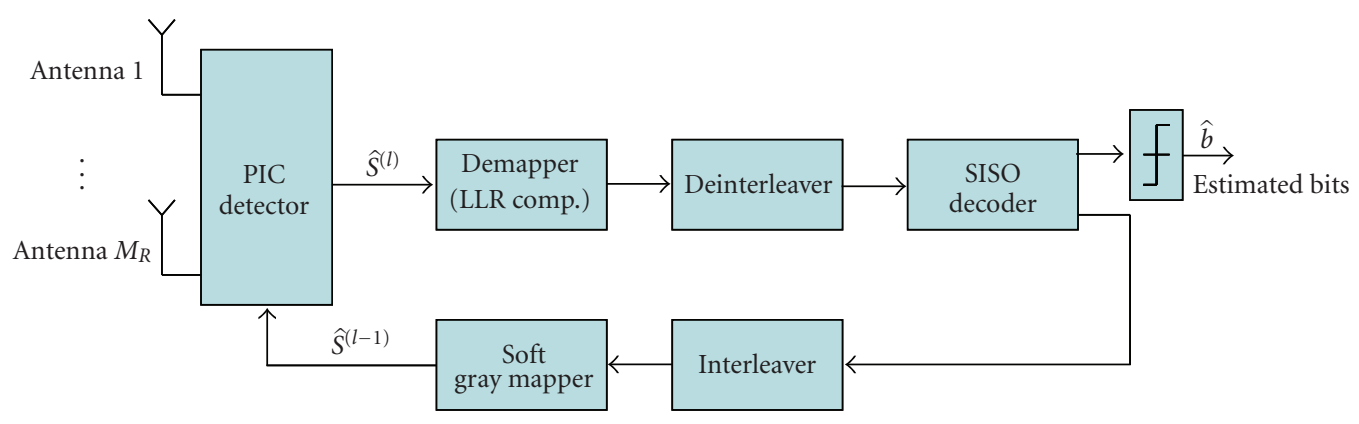

FIGURE 4: Iterative receiver structure.

this case, the maximum likelihood (ML) receiver is simply implemented. This code is described by its dispersion matrix given by

$$
\mathbf{X}=\left[\begin{array}{cc}
s_{1} & s_{2} \\
-s_{2}^{*} & s_{1}^{*}
\end{array}\right]
$$

For NO schemes, we consider in this work the well-known space multiplexing (SM) scheme [13]. SM is designed to maximize the rate by transmitting symbols sequentially on different antennas. Its coding scheme is given by

$$
\mathbf{X}=\left[\begin{array}{ll}
s_{1} & s_{2}
\end{array}\right]^{\mathrm{tr}} .
$$

Finally, we consider the full rate and the fully diverse Golden code [14]. The Golden code is designed to maximize the rate such that the diversity gain is preserved for an increased signal constellation size. It is defined by

$$
\mathbf{X}=\frac{1}{\sqrt{5}}\left[\begin{array}{cc}
\alpha\left(s_{1}+\theta s_{2}\right) & \alpha\left(s_{3}+\theta s_{4}\right) \\
\mu \bar{\alpha}\left(s_{3}+\bar{\theta} s_{4}\right) & \bar{\alpha}\left(s_{1}+\bar{\theta} s_{2}\right)
\end{array}\right],
$$

where $\theta=(1+\sqrt{5}) / 2, \bar{\theta}=1-\theta, \alpha=1+j(1-\theta), \bar{\alpha}=$ $1+j(1-\bar{\theta})$. To identify the most efficient ST code, the OFDM parameters are derived from those of a DVB-T system (see Table 1). Moreover, we have considered the possibility to extend the size of the constellation size up to 256-QAM. The spectral efficiencies 4 and $6 \mathrm{~b} / \mathrm{s} / \mathrm{Hz}$ are obtained for different ST schemes as shown in Table 2. In all simulations, we assume that two $\mathrm{Rx}$ are used by the MT.

In the simulations results given hereafter, we separate the single-layer case and the double-layer case. For NO schemes, we show in [7] that the receiver converges after 3 iterations. This implies an acceptable complexity as compared to the ML detection. This can be observed with Golden code, but also with SM scheme. That is, for NO-STBC schemes, we will present in the sequel the performances after 3 iterations only.

\subsection{Single-layer case: inter-cell ST coding}

In the case of single-layer reception, we have one antenna by site. Then, the second-layer matrix $\mathbf{X}^{(2)}$ in (5) resumes to one element. The multiple-input component of the MIMO scheme is then only obtained by the single antenna in

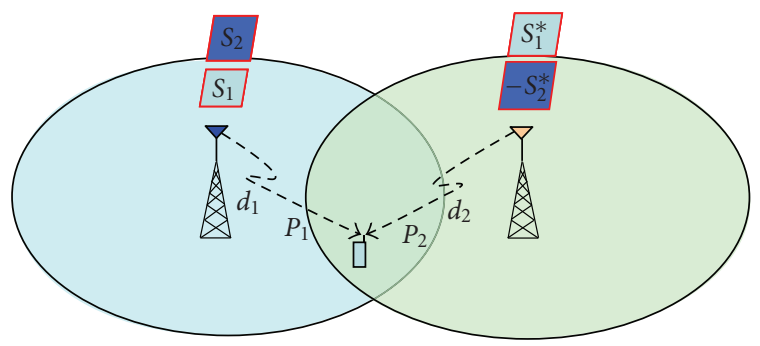

FIgURE 5: Alamouti scheme in SFN environment.

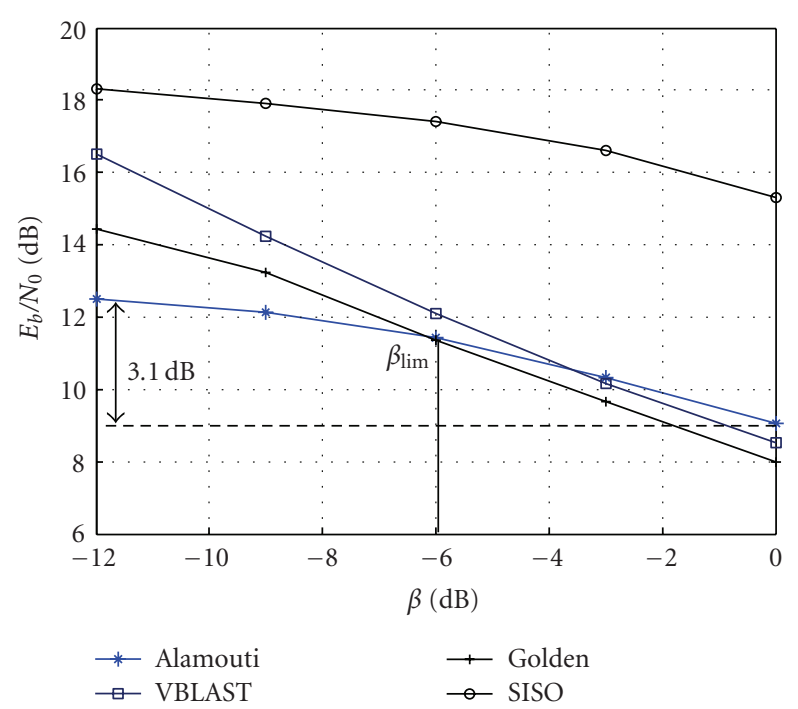

FIgURE 6: Required $E_{b} / N_{0}$ to obtain a BER $=10^{-4}$, single-layer case, $\eta=4 \mathrm{~b} / \mathrm{s} / \mathrm{Hz}$.

each site. Due to the mobility, the MT is assumed to occupy different locations and the first-layer ST scheme must be efficient face to unequal received powers. For equal received powers, we assume that the powers of matrix $\mathbf{B}$ in (14) are equal to $0 \mathrm{~dB}$. Figure 5 presents a simple case of MIMO transmission in SFN environment using Alamouti scheme. As it is shown in this figure, the STBC symbols are transmitted through the set of one antenna in each site using Alamouti coding. 
TABLE 1: Simulations parameters.

\begin{tabular}{ll}
\hline FFT size & $8 \mathrm{~K}$ \\
\hline Sampling frequency $\left(f_{s}=1 / T_{s}\right)$ & $9.14 \mathrm{MHz}$ \\
Guard interval (GI) duration & $1024 \times T_{s}=112 \mu \mathrm{s}$ \\
Rate $R_{c}$ of convolutional code & $1 / 2,2 / 3,3 / 4$ \\
Polynomial code generator & $(133,171)_{\mathrm{o}}$ \\
Channel estimation & perfect \\
Constellation & $16-\mathrm{QAM}, 64-\mathrm{QAM}, 256-\mathrm{QAM}$ \\
Spectral Efficiencies & $\eta=4$ and 6b/s/Hz \\
\hline
\end{tabular}

Figure 6 shows the required $E_{b} / N_{0}$ to obtain a BER equal to $10^{-4}$ for a spectral efficiency $\eta=4 \mathrm{~b} / \mathrm{s} / \mathrm{Hz}$ with a Rayleigh channel model. Since we have one Tx antenna by site, we set $\beta_{1}=0 \mathrm{~dB}$ and we change $\beta=\beta_{2}$. As expected, this figure shows that the Golden code presents the best performance when the Rx receives the same power from both sites (i.e., $\beta_{1}=\beta_{2}=0 \mathrm{~dB}$ ). When $\beta_{2}$ decreases, Alamouti scheme is very efficient and presents a maximum loss of only $3 \mathrm{~dB}$ in terms of required $E_{b} / N_{0}$ with respect to equal received powers case. Indeed, for very small values of $\beta$, the transmission scenario becomes equivalent to a scenario with one transmitting antenna. In this figure, the value $\beta_{\lim }=-6.2 \mathrm{~dB}$ presents the power imbalance limit where the Alamouti and the Golden code schemes have the same performance at a $\mathrm{BER}=10^{-4}$. It is also straightforward to note that the SISO transmission in existing SFN presents the worst results when it is compared to the distributed MIMO technique.

\subsection{Double-layer case}

Considering the whole double-layer space domain construction, one ST coding scheme has to be assigned to each layer of the proposed system. The resulting 3D STS code should be efficient for both environments in SFN architectures. In this paper, we restrict our study to $M_{T}=2 \mathrm{Tx}$ by site. We propose to construct the first layer with Alamouti scheme, since it is the most resistant for the unequal received powers case. In a complementary way, we propose to construct the second layer with the Golden code since it offers the best results in the case of equal received powers. After combination of the two space layers with time dimension, (5) yields

$$
X=1 / \sqrt{5}\left(\begin{array}{cccc}
\alpha\left(s_{1}+\theta s_{2}\right) & \alpha\left(s_{3}+\theta s_{4}\right) & \alpha\left(s_{5}+\theta s_{6}\right) & \alpha\left(s_{7}+\theta s_{8}\right) \\
j \bar{\alpha}\left(s_{3}+\bar{\theta} s_{4}\right) & \bar{\alpha}\left(s_{1}+\bar{\theta} s_{2}\right) & j \bar{\alpha}\left(s_{7}+\bar{\theta} s_{8}\right) & \bar{\alpha}\left(s_{5}+\bar{\theta} s_{6}\right) \\
-\alpha^{*}\left(s_{5}^{*}+\theta^{*} s_{6}^{*}\right) & -\alpha^{*}\left(s_{7}^{*}+\theta^{*} s_{8}^{*}\right) & \alpha^{*}\left(s_{1}^{*}+\theta^{*} s_{2}^{*}\right) & \alpha^{*}\left(s_{3}^{*}+\theta^{*} s_{4}^{*}\right) \\
j \bar{\alpha}^{*}\left(s_{7}^{*}+\overline{\theta^{*}} s_{8}^{*}\right) & -\overline{\alpha^{*}}\left(s_{5}^{*}+\overline{\left.\theta^{*} s_{6}^{*}\right)}\right. & -j \overline{\alpha^{*}}\left(s_{3}^{*}+\overline{\theta^{*}} s_{4}^{*}\right) & \alpha^{*}\left(s_{1}^{*}+\overline{\theta^{*}} s_{2}^{*}\right)
\end{array}\right)
$$

where $\theta=(1+\sqrt{5}) / 2, \bar{\theta}=1-\theta, \alpha=1+j(1-\theta), \bar{\alpha}=$ $1+j(1-\bar{\theta})$.

Since the distance $d$ between the transmitting antennas in one site is negligible with respect to the distance $D$ (Figure 1), the power attenuation factors in the case of our $3 \mathrm{D}$ code are such that $\beta_{1}=\beta_{2}=0 \mathrm{~dB}$ and $\beta=\beta_{3}=\beta_{4}$. Figure 7 presents an overview of the proposed 3D STSBC. Since the received powers from each antenna in the same site are equal, we apply the Golden code between the two signals transmitted in a given site. However, we apply the Alamouti code between the signals transmitted by the different sites' antennas.

Figure 8 shows the results in terms of required $E_{b} / N_{0}$ to obtain a BER equal to $10^{-4}$ for different values of $\beta$ and 3 STBC schemes, that is, our proposed 3D code scheme, the single-layer Alamouti scheme, and the single-layer Golden scheme. The results obtained in this figure assume that the transmission is achieved through the COST 207 TU-6 channel model [15]. The value $\beta$ in this figure corresponds to $\beta_{2}$ for the single-layer case and to $\beta=\beta_{3}=\beta_{4}$ for our $3 \mathrm{D}$ code. We assume that the MT is moving with a velocity of $10 \mathrm{~km} / \mathrm{h}$ and the distance $d_{1}$ of the reference antenna is equal to $5 \mathrm{~km}$. The CIRs between different transmitters and the MT are delayed according to (3). Figure 8 shows that the proposed scheme presents the best performance whatever the spectral efficiency and the factor $\beta$ are. Indeed, it is optimized for SFN systems and unbalanced received powers. For $\beta=-12 \mathrm{~dB}$, the proposed 3D code offers a gain equal to $1.5 \mathrm{~dB}$ (resp., $3.1 \mathrm{~dB}$ ) with respect to the Alamouti scheme

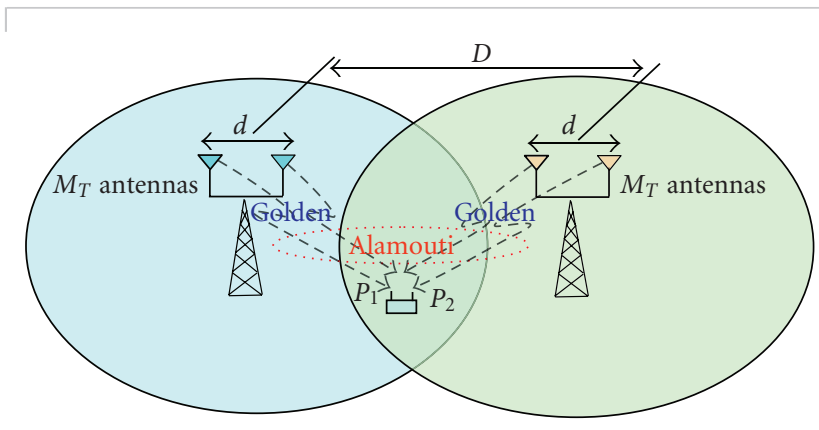

FIGURE 7: 3D STS scheme in SFN environment.

for a spectral efficiency $\eta=4 \mathrm{~b} / \mathrm{s} / \mathrm{Hz}$ (resp. $\eta=6 \mathrm{~b} / \mathrm{s} / \mathrm{Hz}$ ). This gain is greater when it is compared to the Golden code. The maximum loss of our code due to unbalanced received powers is equal to $3 \mathrm{~dB}$ in terms of $E_{b} / N_{0}$. This means that it leads to a powerful code for SFN systems.

Figure 9 evaluates the robustness of the different schemes to the two values of MT velocity, that is, $10 \mathrm{~km} / \mathrm{h}$ and $60 \mathrm{~km} / \mathrm{h}$. We show in this figure that the Alamouti scheme is very robust to the MT velocity. The degradation of the Golden code might reach $1 \mathrm{~dB}$ in terms of required $E_{b} / N_{0}$ at a $\mathrm{BER}=10^{-4}$. Our $3 \mathrm{D}$ code presents an intermediate behaviour. Its degradation due to the MT velocity, that is, to Doppler effect is about $0.2 \mathrm{~dB}$ only. 
TABle 2: Different MIMO schemes and efficiencies.

\begin{tabular}{|c|c|c|c|c|}
\hline Spectral efficiency & ST scheme & ST rate $R$ & Constellation & $R_{c}$ \\
\hline \multirow{4}{*}{$\eta=4[\mathrm{bit} / \mathrm{Sec} / \mathrm{Hz}]$} & Alamouti & 1 & 64-QAM & $2 / 3$ \\
\hline & SM & 2 & 16-QAM & $1 / 2$ \\
\hline & Golden & 2 & 16-QAM & $1 / 2$ \\
\hline & 3D code & 2 & 16-QAM & $1 / 2$ \\
\hline \multirow{4}{*}{$\eta=6[\mathrm{bit} / \mathrm{Sec} / \mathrm{Hz}]$} & Alamouti & 1 & 256-QAM & $3 / 4$ \\
\hline & SM & 2 & 64-QAM & $1 / 2$ \\
\hline & Golden & 2 & 64-QAM & $1 / 2$ \\
\hline & 3D code & 2 & 64-QAM & $1 / 2$ \\
\hline
\end{tabular}

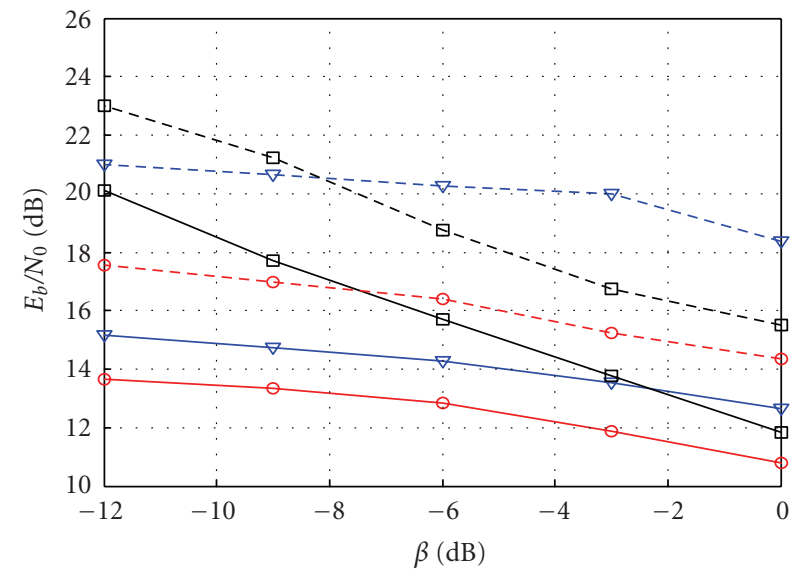

$$
\begin{array}{ll}
\rightarrow \text { BD code } \eta=4 \mathrm{~b} / \mathrm{s} / \mathrm{Hz} & -\ominus-3 \mathrm{D} \text { code } \eta=6 \mathrm{~b} / \mathrm{s} / \mathrm{Hz} \\
\rightarrow \text { Alamouti } \eta=4 \mathrm{~b} / \mathrm{s} / \mathrm{Hz} & -\nabla-\text { Alamouti } \eta=6 \mathrm{~b} / \mathrm{s} / \mathrm{Hz} \\
\square-\text { Golden } \eta=4 \mathrm{~b} / \mathrm{s} / \mathrm{Hz} & -\bullet-\text { Golden } \eta=6 \mathrm{~b} / \mathrm{s} / \mathrm{Hz}
\end{array}
$$

FIGURE 8: Required $E_{b} / N_{0}$ to obtain a BER $=10^{-4}$, double-layer case, $\eta=4 \mathrm{~b} / \mathrm{s} / \mathrm{Hz}, \eta=6 \mathrm{~b} / \mathrm{s} / \mathrm{Hz}$, TU-6 channel.

\subsection{Complexity considerations}

As we have shown, the $3 \mathrm{D}$ code outperforms the other MIMO schemes in different reception scenarios. Let us now compare the different MIMO schemes in terms of complexity implementation. At this stage, different complexity points could be evaluated. First, at the transmission side, the implementation of the Alamouti and the Golden code schemes between different sites in SFN architecture does not increase the complexity when it is compared to that of the SISO case. Indeed, we just need to synchronize the transmission from both sites as it should be already done with SFN in the SISO case. This task can be ensured by an ultrastable reference like the GPS. However, for the 3D code, an additional front-end RF should be used at each site. At the receiving side, the iterative receiver used for NO schemes like the SM scheme or the Golden code is the same of that used for the 3D code. Moreover, when compared to the ML detection, we had showed in [7] that the iterative receiver converges after 3 iterations only. However, the ML detection complexity increases with the MIMO architecture size and the modulation order [16]. Thus, the proposed 3D code complexity is of the same order of the NO codes complexity.

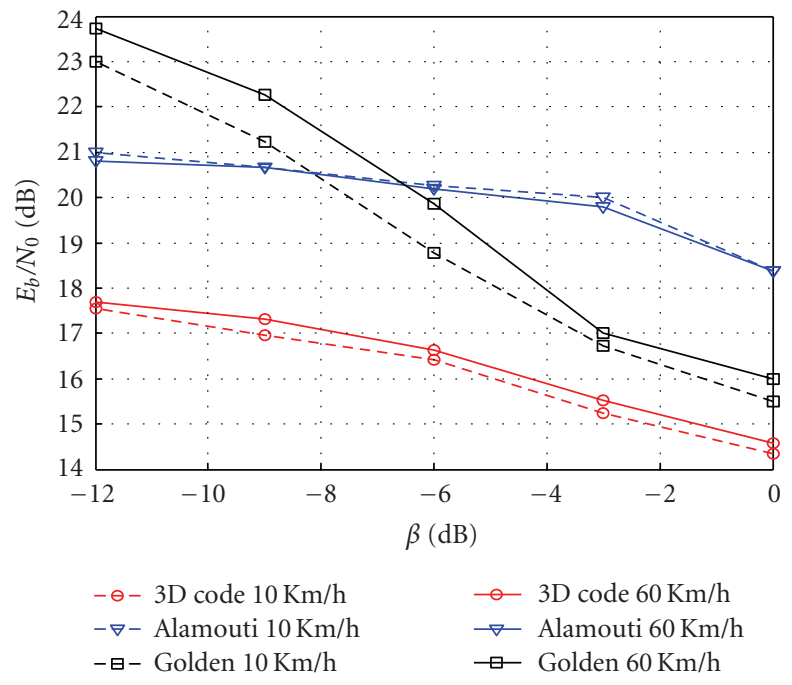

FIgURE 9: Required $E_{b} / N_{0}$ to obtain a BER $=10^{-4}, \eta=6 \mathrm{~b} / \mathrm{s} / \mathrm{Hz}$, TU-6 channel, different values of MT velocity.

\section{ANALYTICAL SYSTEM LEVEL EVALUATION}

In the previous section, we have proposed a new 3D STSBC for MIMO-OFDM systems in SFN architecture. Using the system level simulations, we have showed that this new ST code is very efficient to cope with equal and unequal received powers. However, explicit bit level simulation of each MT in every cell of the SFN would be forbidding time consuming. The problem becomes more noticeable when MIMO-OFDM techniques are used in SFN architectures. As a consequence, it is desirable to evaluate the system level performance in terms of BER without achieving system simulation. Thus, the practical need of an accurate abstraction of the system level simulation into analytical evaluation highly motivates our work to achieve an analytical BER expression of the MIMOOFDM systems using iterative receiver.

In some studies, it has been shown that the BER at the output of the channel decoder is directly related to the SINR at the output of the detector $[8,17,18]$. In the OFDM system, [8] proposes a new technique called effective exponential SINR mapping (EESM) to evaluate the BER. The technique is based on the computation of an effective SINR derived from the different values of the estimated SINRs on each 
subcarrier. The authors of [19] propose a new method to adapt the EESM technique to the SM transmission and an ML receiver. Their method, however, could not be suited for our work since an iterative receiver is used and, as it will be shown, the SNR expression is not computed for each layer.

In this paper, we propose to adapt the EESM technique to the MIMO-OFDM systems using the iterative receiver. The first step in our work consists in computing the SINRs expressions at the output of the detector. The second step is to establish an accurate relationship between the different SINRs and the coded BER through the adapted EESM technique. We note that the SINR expressions and the predicted BER given hereafter are not specified for a given antenna, that is, we do not separate between the different antennas like in [19]. However, it is possible to apply our methods for each antenna received signal.

\subsection{SINR evaluation}

Without loss of generality, we assume in the sequel that we are interested by the $p$ th symbol. Using the vector-matrix notation of Sections 4 and 5, the estimated received symbol at the first iteration in (18) could be written in an equivalent form as

$$
\begin{gathered}
\hat{s}_{p}=I_{0}^{(1)}+I_{1}^{(1)}+I_{2}^{(1)}, \\
I_{0}^{(1)}=\mathbf{g}_{\mathbf{p}}^{\operatorname{tr}}\left(\mathbf{G}_{\mathbf{e q}} \cdot \mathbf{G}_{\mathbf{e q}}^{\operatorname{tr}}+\sigma_{w}^{2} \mathbf{I}\right)^{-1} \mathbf{g}_{\mathbf{p}} s_{p}, \\
I_{1}^{(1)}=\sum_{\substack{q=1 \\
q \neq p}}^{2 Q} \mathbf{g}_{\mathbf{p}}^{\operatorname{tr}}\left(\mathbf{G}_{\mathbf{e q}} \cdot \mathbf{G}_{\mathbf{e q}}^{\operatorname{tr}}+\sigma_{w}^{2} \mathbf{I}\right)^{-1} \mathbf{g}_{q} s_{q}, \\
I_{2}^{(1)}=\mathbf{g}_{\mathbf{p}}^{\operatorname{tr}}\left(\mathbf{G}_{\mathbf{e q}} \cdot \mathbf{G}_{\mathbf{e q}}^{\operatorname{tr}}+\sigma_{w}^{2} \mathbf{I}\right)^{-1} \mathbf{w} .
\end{gathered}
$$

In (24), $I_{0}^{(1)}$ is the useful received signal, $I_{1}^{(1)}$ is the IEI due to the nonorthogonality of the considered STBC. We can easily verify that it is equal to zero for O-STBC schemes. $I_{2}^{(1)}$ is the coloured noise. The superscript $\ell$ in the signal expressions $I^{(\ell)}$ indicates the number of iteration in the iterative process.

The complex transmitted data symbols are assumed i.i.d. having zero mean and unit variance (the variance of the real and imaginary parts is equal to $1 / 2$ ). Due to this distribution, the SINR expression can be deduced from (24) by

$$
\operatorname{SINR}=\frac{E\left\{\left|I_{0}^{(1)}\right|^{2}\right\}}{E\left\{\left|I_{1}^{(1)}\right|^{2}\right\}+E\left\{\left|I_{2}^{(1)}\right|^{2}\right\}} .
$$

The expectations values in (25) over the random data symbols are given by

$$
\begin{aligned}
& E\left\{\left|I_{0}^{(1)}\right|^{2}\right\}=\frac{1}{2}\left|\mathbf{g}_{\mathbf{p}}^{\operatorname{tr}}\left(\mathbf{G}_{\mathbf{e q}} \cdot \mathbf{G}_{\mathbf{e q}}^{\mathrm{tr}}+\sigma_{w}^{2} \mathbf{I}\right)^{-1} \mathbf{g}_{\mathbf{p}}\right|^{2}, \\
& E\left\{\left|I_{1}^{(1)}\right|^{2}\right\}=\frac{1}{2} \sum_{\substack{q=1 \\
q \neq p}}^{2 Q}\left|\mathbf{g}_{\mathbf{p}}^{\mathrm{tr}}\left(\mathbf{G}_{\mathbf{e q}} \cdot \mathbf{G}_{\mathbf{e q}}^{\mathrm{tr}}+\sigma_{w}^{2} \mathbf{I}\right)^{-1} \mathbf{g}_{\mathbf{q}}\right|^{2}, \\
& E\left\{\left|I_{2}^{(1)}\right|^{2}\right\}=\frac{N_{0}}{2}\left|\mathbf{g}_{\mathbf{p}}^{\operatorname{tr}}\left(\mathbf{G}_{\mathbf{e q}} \cdot \mathbf{G}_{\mathbf{e q}}^{\mathrm{tr}}+\sigma_{w}^{2} \mathbf{I}\right)^{-1}\right|^{2} .
\end{aligned}
$$

At the second iteration, the estimated symbol expressed in (24) becomes more complex. It is obtained using (17) and (18) in (19) by

$$
\widehat{s}_{p}^{(2)}=I_{0}^{(2)}+I_{1}^{(2)}+I_{2}^{(2)}
$$

where

$$
\begin{gathered}
I_{0}^{(2)}=s_{p}, \\
I_{1}^{(2)}=\sum_{\substack{q=1 \\
q \neq p}}^{2 Q} \frac{1}{\mathbf{g}_{\mathbf{p}}^{\operatorname{tr}} \mathbf{g}_{\mathbf{p}}} \mathbf{g}_{\mathbf{p}}^{\operatorname{tr}}\left[\mathbf{g}_{\mathbf{q}} s_{q}-\left(\mathbf{G}_{\mathbf{e q}} \cdot \mathbf{G}_{\mathbf{e q}}^{\operatorname{tr}}+\sigma_{w}^{2} \mathbf{I}\right)^{-1} \mathbf{g}_{\mathbf{q}} \tilde{s}_{q}^{(1)}\right], \\
I_{2}^{(2)}=\frac{1}{\mathbf{g}_{\mathbf{p}}^{\operatorname{tr}} \mathbf{g}_{\mathbf{p}}} \mathbf{g}_{\mathbf{p}}^{\operatorname{tr}} \mathbf{w} .
\end{gathered}
$$

For next iterations, it is clear from (27) that the expressions of the estimated received symbol as well as the estimated SINR become more complex. Therefore, some manipulations should be considered to give an analytical expression of the SINR.

Based on the structure of the iterative receiver, we already know that the outputs of the soft Gray mapper are complex symbols which belong to the set of constellation points. Let $I_{t}^{(2)}=I_{1}^{(2)}+I_{2}^{(2)}=s_{p}^{(2)}-I_{0}^{(2)}$ be the total interference power at the second iteration. Then, two cases can be presented at this stage.

(I) If the estimated symbol $\widetilde{s}_{p}^{(1)}$ at the output of the Gray mapper is equal to the transmitted symbol $s_{p}$, the useful signal $I_{0}^{(2)}$ in (28) is such that $I_{0}^{(2)}=s_{p}=\tilde{s}_{p}^{(1)}$ and the total interference signal at the second iteration becomes

$$
I_{t}^{(2)}=I_{1}^{(2)}+I_{2}^{(2)}=s_{p}^{(2)}-s_{p}^{(1)}
$$

Since $I_{1}^{(2)}$ and $I_{2}^{(2)}$ are independent and the complex outputs of the Gray mapper are zero mean with unit variance, the estimated SINR at the second iteration is

$$
\operatorname{SINR}^{(2)}=\frac{E\left\{\left|I_{0}^{(2)}\right|^{2}\right\}}{E\left\{\left|I_{1}^{(2)}+I_{2}^{(2)}\right|^{2}\right\}}=\frac{1}{2 \cdot E\left\{\left|\hat{s}_{p}^{(2)}-\tilde{s}_{p}^{(1)}\right|^{2}\right\}}
$$

where $\hat{s}_{p}^{(2)}$ is estimated through (19) and $\widetilde{s}_{p}^{(1)}$ is the output of the soft Gray mapper at the first iteration.

(II) If the estimated symbol $\tilde{s}_{p}^{(1)}$ at the first iteration is different from the transmitted symbol $s_{p}$, the difference between the received signals at the first two successive iterations yields by substituting $I_{0}^{(2)}$ from (28) in (27):

$$
\hat{s}_{p}^{(2)}-\widetilde{s}_{p}^{(1)}=s_{p}+I_{1}^{(2)}+I_{2}^{(2)}-\widetilde{s}_{p}^{(1)}
$$


Since $\widetilde{s}_{p}^{(1)}$ is different from $s_{p}$ in this case, and the different transmitted symbols are i.i.d., we can verify due to the expectation operation that

$$
\begin{aligned}
\operatorname{SINR}^{(2)} & =\frac{E\left\{\left|I_{0}^{(2)}\right|^{2}\right\}}{E\left\{\left|I_{1}^{(2)}+I_{2}^{(2)}\right|^{2}\right\}} \\
& =\frac{E\left\{\left|I_{0}^{(2)}\right|^{2}\right\}}{E\left\{\left|I_{1}^{(2)}+I_{2}^{(2)}+s_{p}-\widetilde{s}_{p}^{(1)}\right|^{2}\right\}} \\
& =\frac{E\left\{\left|I_{0}\right|^{2}\right\}}{E\left\{\left|\hat{s}_{p}^{(2)}-\widetilde{s}_{p}^{(1)}\right|^{2}\right\}} \\
& =\frac{1}{2 \cdot E\left\{\left|\hat{s}_{p}^{(2)}-\tilde{s}_{p}^{(1)}\right|^{2}\right\}} .
\end{aligned}
$$

It is clear from the last term of (30) and (32) that the SINR expression at the second iteration is simpler than that of (28). In this case, only the estimated symbols at each iteration are used for SINR estimation, that is, we do not have to compute complex expressions. Also, we can show that (30) and (32) could be generalized for successive iterations. In the next section, we will exploit our theoretical SINR model through BER measurements at the output of the channel decoder.

\subsection{BER evaluation with EESM technique}

In order to evaluate the BER at the output of the channel decoder, we propose in this section to adapt the EESM technique to the MIMO-OFDM context. At the first step, we will develop analytically the EESM technique. At the second step, we will present its application in the OFDM system. Then, we will adapt it to the MIMO-OFDM context using the SINR expressions computed at the previous subsection.

Let $J$ denote the packet size in complex data symbols. In general, the data symbols in the packets are transmitted over different resource elements (e.g., subcarriers) and, therefore, they may experience different propagation and interference conditions. Thus, the data symbols may have different SINR values. Let SINR be the vector of $J$ instantaneous SINR received at the output of the detector. The problem of determining an accurate BER prediction method comes back to looking for a relationship such that

$$
P_{e}=f(\operatorname{SINR})
$$

where $P_{e}$ denotes the bit error probability (BEP) and $f$ is the prediction function, which should be invariant with respect to the fading realization and to the multipath channel model, and should be applicable to different MCSs in a soft way, that is, by changing the values of some generic parameters [18]. In the context of AWGN channel, the SINR becomes SNR and it remains constant over the packet. In this context, a direct relationship $\xi$ exists between the SNR and the error probability:

$$
P_{e, W G N}=\xi(\mathrm{SNR})
$$

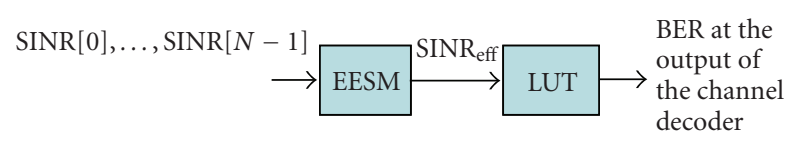

FIgURE 10: BER prediction through EESM.

The function $\xi$ is called the mapping function. It is obtained through theoretical analysis or system level simulation with AWGN channel. In the general context of a fading channel, where the SINR varies, the function $f$ in (33) can be written exactly as a compound function of the AWGN function $\xi$ and a compression function $r$ [8]:

$$
\begin{gathered}
P_{e}=\xi \text { o } r(\mathrm{SINR})=\xi\left(\mathrm{SINR}_{\mathrm{eff}}\right) \\
\text { with } \mathrm{SINR}_{\mathrm{eff}}=r(\mathrm{SINR}) .
\end{gathered}
$$

The function $r$ is referred to as the compression functionsince its role is to compress the vector SINR of $J$ components

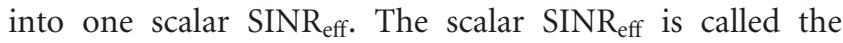
effective SINR and it is defined as the SINR which would yield the same error probability in an equivalent AWGN channel as the associated vector SINR in a fading channel. By writing (35), we have merely turned the problem of determining the evaluation function $f$ into the problem of determining the compression function $r$.

In an OFDM system, it was concluded that the key issue to accurately determine the appropriate BER after channel decoding is to use the effective SINR in combination with AWGN curves. The work in [8] proposes the EESM technique which is based on the Chernoff Union bound [18] to find the effective SINR. The key EESM technique expression relevant to an OFDM system is given by

$$
\mathrm{SINR}_{\mathrm{eff}}=-\lambda \ln \left(\frac{1}{N} \sum_{n=0}^{N-1} \exp \left(-\frac{\operatorname{SINR}[n]}{\lambda}\right)\right) .
$$

SINR $[n]$ is the SINR obtained over the $n$th sub-carrier and $\lambda$ is a unique parameter which must be estimated from the system level simulations for each MCS. It is estimated once by preliminary simulation for each MCS. When the SINR eff is computed, it will be used for BER prediction at the output of the channel decoder with a simple lookup table (LUT) as shown in Figure 10. This LUT gives the BER at the output of the channel decoder as a function of the SNR for a Gaussian channel. It is computed analytically or by simulations. The uniqueness of $\lambda$ for each MCS is derived from the fact that the effective SINR must fulfil the approximate relation

$$
\operatorname{BER}(\operatorname{SINR})=\mathrm{BER}_{\mathrm{AWGN}}\left(\mathrm{SINR}_{\mathrm{eff}}\right) \text {, }
$$

where $B R_{A W G N}$ is the BEP for the AWGN channel which depends only on the MCSs.

In our study, the EESM technique must be adapted to the MIMO-OFDM system. Indeed, the estimated received symbol at each subcarrier is a superposition of different symbols transmitted by the different antennas on that subcarrier. Therefore, the EESM technique will be applied on the set of $\mathrm{Q}$ symbols transmitted on the $M_{T}$ antennas 


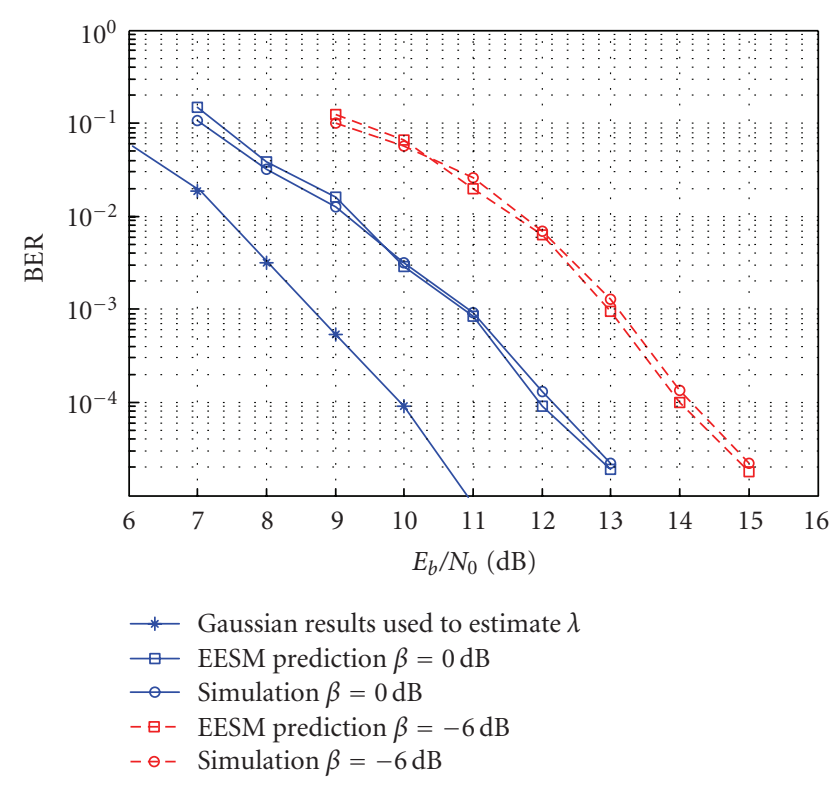

FIgURE 11: Validation of EESM technique, Alamouti scheme, $\eta=$ $6 \mathrm{~b} / \mathrm{s} / \mathrm{Hz}, \lambda=22.6$, TU-6 channel.

during $T$ OFDM symbols. The effective SINR is, therefore, computed through

$$
\operatorname{SINR}_{\mathrm{eff}}=-\lambda \ln \left(\frac{1}{N Q} \sum_{q=1}^{Q} \sum_{n=1}^{N} \exp \left(-\frac{\operatorname{SINR}_{q}[n]}{\lambda}\right)\right) .
$$

Using the effective SINR of (38), we are now able to evaluate the BER using the LUT as shown in Figure 10.

\section{APPLICATION OF THE EESM TECHNIQUE TO THE PROPOSED 3D STS CODE}

In this section, we validate through the EESM technique and the SINR analysis the efficiency of the proposed 3D STS code. The considered simulation parameters are the same of those given in Table 1. The parameter $\lambda$ is estimated using the AWGN channel model. Its estimation is done as follows. For a given channel model and a given MCS, we estimate the different SINRs at the output of the detector. Therefore, we select a value of $\lambda$ and we compute the BER at the output of the channel decoder according to (38) and Figure 10. The accurate value of $\lambda$ is that which ensures minimum error between the predicted and the simulated BERs. Once the value of $\lambda$ is decided, it does not change with the channel or the MIMO scheme. We will show by simulations that this value depends only on the spectral efficiency.

The results given in this section are obtained with the COST 207 TU-6 channel model. The AWGN results used to estimate the parameter $\lambda$ are obtained using Alamouti scheme since NO schemes are not efficient with AWGN channel. They will be plotted in the same figure of those obtained by EESM technique or by simulations, with TU6 channel. First of all, we will validate our SINR analysis and EESM technique on the Alamouti and the Golden code schemes. Then, it will be suited by the validation of the

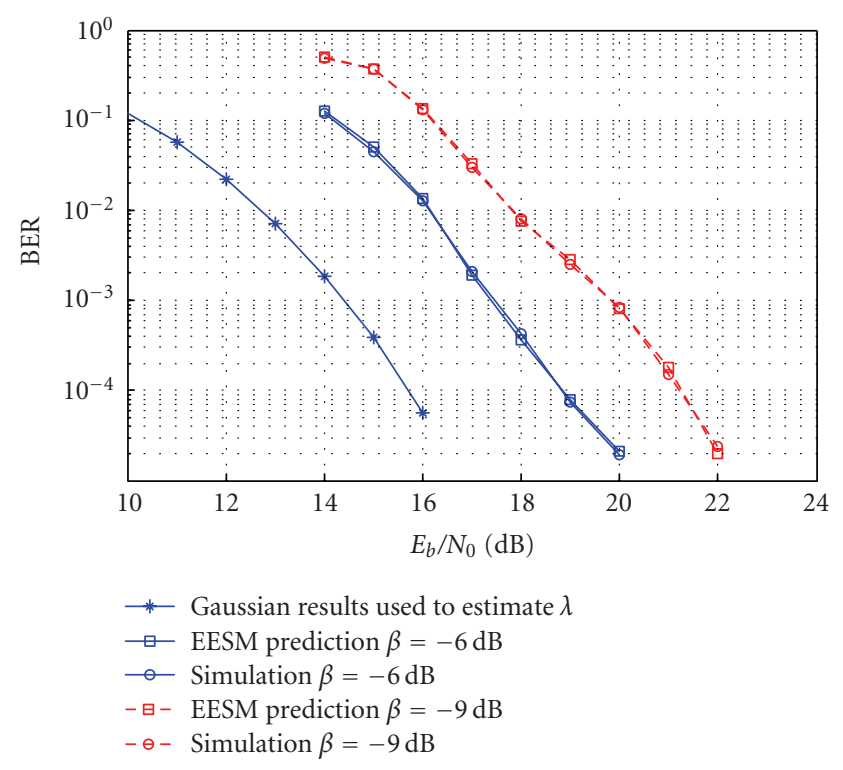

FIgUre 12: Validation of EESM technique, Golden code scheme, $\eta=6 \mathrm{~b} / \mathrm{s} / \mathrm{Hz}, \lambda=22.6$, TU-6 channel.

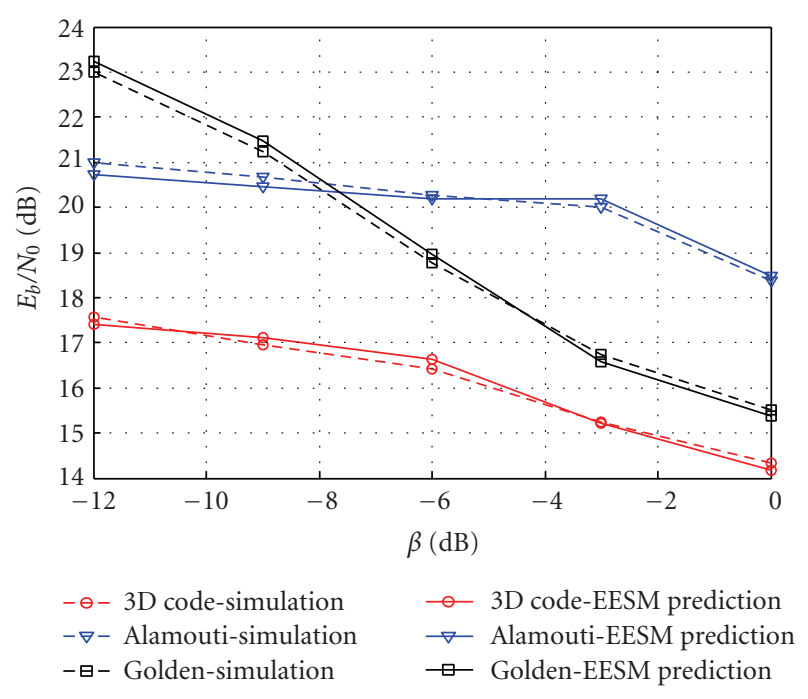

Figure 13: Validation of EESM technique, $\eta=6 \mathrm{~b} / \mathrm{s} / \mathrm{Hz}, \lambda=22.6$, TU-6 channel.

efficiency of our proposed code. In all figures, the results are given with mobile velocity equal to $10 \mathrm{~km} / \mathrm{h}$.

Figure 11 compares the BER obtained by simulations and the BER obtained with the EESM technique for the Alamouti scheme, considering a spectral efficiency $\eta=$ $6 \mathrm{~b} / \mathrm{s} / \mathrm{Hz}$ and different values of transmitted powers. These figures show the accuracy of the proposed technique based on the SINR analytical expression. Moreover, they show that the parameter $\lambda$ is constant $(\lambda=22.6$ for $\eta=6)$ and it is independent of the power imbalance but depends on the MCSs or equivalently on the spectral efficiency. The parameter $\lambda$ is obtained by simulations. It is computed once for a given MCS. 
Figure 12 compares the BER obtained by simulations and the BER evaluated with the EESM technique for the Golden code scheme, a spectral efficiency $\eta=6 \mathrm{~b} / \mathrm{s} / \mathrm{Hz}$ and different values of transmitted powers. Since, compared with the parameters used in Figure 11, the spectral efficiency does not change, the parameter $\lambda=22.6$ gives an accurate BER prediction and validates our analytical expressions and prediction method. Again, we show that this parameter is independent of the power imbalance.

In Figure 13, we give the same kind of results of those given in Figure 8 by using the EESM technique. Figure 13 validates our proposed prediction method for all considered STBC schemes in the SFN architecture. Again, it shows that parameter $\lambda$ is independent of the power imbalance and of the STBC scheme. Moreover, it shows again the superiority of the proposed 3D STSBC whatever the power imbalance factor $\beta$.

\section{CONCLUSION}

In this paper, a new 3D STSBC has been presented for MIMO transmission in SFN architecture including two transmitting sites. The proposed 3D STSBC is based on a double-layer structure defined for intercell and intracell situations by adequately combining the Alamouti code and the Golden code schemes. We showed that our proposed 3D STS scheme is very efficient to cope with equal and unequal received powers in SFN scenarios whatever the receiver position is.

Moreover, we have proposed an analytical SINR evaluation of MIMO-OFDM systems using an iterative receiver as well as an adaptation of the EESM technique to efficiently evaluate the BER at the output of the channel decoder. Using the EESM technique and the analytical evaluation, we have showed again the superiority of the proposed $3 \mathrm{D}$ code. It is then a very promising candidate for the broadcasting of the future terrestrial digital TV in SFN architectures.

\section{ACKNOWLEDGMENT}

The authors would like to thank the European CELTIC Project "B21C" for its support to this work.

\section{REFERENCES}

[1] A. Mattsson, "Single frequency networks in DTV," IEEE Transactions on Broadcasting, vol. 51, no. 4, pp. 413-422, 2005.

[2] L. Zhang, L. Gui, Y. Qiao, and W. Zhang, "Obtaining diversity gain for DTV by using MIMO structure in SFN," IEEE Transactions on Broadcasting, vol. 50, no. 1, pp. 83-90, 2004.

[3] Y. Kanbe, M. Itami, K. Itoh, and A. Hamid Aghvami, "Reception of OFDM signals with an array antenna in a SFN environment," in Proceedings of the 13th IEEE International Symposium on Personal, Indoor and Mobile Radio Communications (PIMRC '02), vol. 3, pp. 1310-1315, Lisbon, Portugal, September 2002.

[4] http://www.celtic-initiative.org/Projects/B21C.

[5] M.-A. Khalighi, J.-F. Hélard, and S. Bourennane, "Contrasting orthogonal and non-orthogonal space-time schemes for perfectly-known and estimated MIMO channels," in Proceedings of the 10th IEEE Singapore International Conference on Communications Systems (ICCS '06), Singapore, October 2006.

[6] Y. Nasser, J.-F. Hélard, and M. Crussiere, "Robustness of MIMO-OFDM schemes for future digital TV to carrier frequency offset," in Proceedings of IEEE International Symposium on Broadband Multimedia Systems and Broadcasting (BMSB '08), pp. 1-4, Las Vegas, Nev, USA, March-April 2008.

[7] Y. Nasser, J.-F. Hélard, M. Crussiere, and O. Pasquero, "Efficient MIMO-OFDM schemes for future terrestrial digital TV with unequal received powers," in Proceedings of IEEE International Conference on Communications (ICC '08), pp. 2021-2027, Bejing, China, May 2008.

[8] 3GPP TSG-RAN-1, “TR 25.892: feasibility study for OFDM for UTRAN enhancement," version 1.1.0, March 2004.

[9] T. A. Lamahewa, R. A. Kennedy, T. D. Abhayapala, and T. Betlehem, "MIMO channel correlation in general scattering environments," in Proceedings of the 7th Australian Communications Theory Workshop (AUSCTW '06), pp. 93-98, Perth, Australia, February 2006.

[10] F. Tosato and P. Bisaglia, "Simplified soft-output demapper for binary interleaved COFDM with application to HIPERLAN/2," in Proceedings of IEEE International Conference on Communications (ICC'02), vol. 2, pp. 664-668, New York, NY, USA, April-May 2002.

[11] J. Hagenauer and P. Hoeher, "A Viterbi algorithm with soft-decision outputs and its applications," in Proceedings of IEEE Global Telecommunications Conference and Exhibition (GLOBECOM '89), vol. 3, pp. 1680-1686, Dallas, Tex, USA, November 1989.

[12] S. M. Alamouti, "A simple transmit diversity technique for wireless communications," IEEE Journal on Selected Areas in Communications, vol. 16, no. 8, pp. 1451-1458, 1998.

[13] G. J. Foschini, "Layered space-time architecture for wireless communication in a fading environment when using multielement antennas," Bell Labs Technical Journal, vol. 1, no. 2, pp. 41-59, 1996.

[14] J.-C. Belfiore, G. Rekaya, and E. Viterbo, "The golden code: a $2 \times 2$ full-rate space-time code with nonvanishing determinants," IEEE Transactions on Information Theory, vol. 51, no. 4, pp. 1432-1436, 2005.

[15] COST 207 Report, "Digital land mobile radio communications, Commission of European Communities, Directorate General, Telecommunications Information Industries and Innovation," Luxemburg, 1989.

[16] M. Rupp, G. Gritsch, and H. Weinrichter, "Approximate ML detection for MIMO systems with very low complexity," in Proceedings of IEEE International Conference on Acoustics, Speech, and Signal Processing (ICASSP '04), vol. 4, pp. 809-812, Montreal, Canada, May 2004.

[17] R. Ratasuk, A. Ghosh, and B. Classon, "Quasi-static method for predicting link-level performance," in Proceedings of the 55th IEEE Vehicular Technology Conference (VTC '02), vol. 3, pp. 1298-1302, Birmingham, Ala, USA, May 2002.

[18] Ericsson, "System level evaluation of OFDM- further considerations," TSG-RAN WG1 \#35, November 2003, R1-031303, Lisbon, Portugal.

[19] H. Liu, L. Cia, H. Yang, and D. Li, "EESM based link error prediction for adaptive MIMO-OFDM system”. 

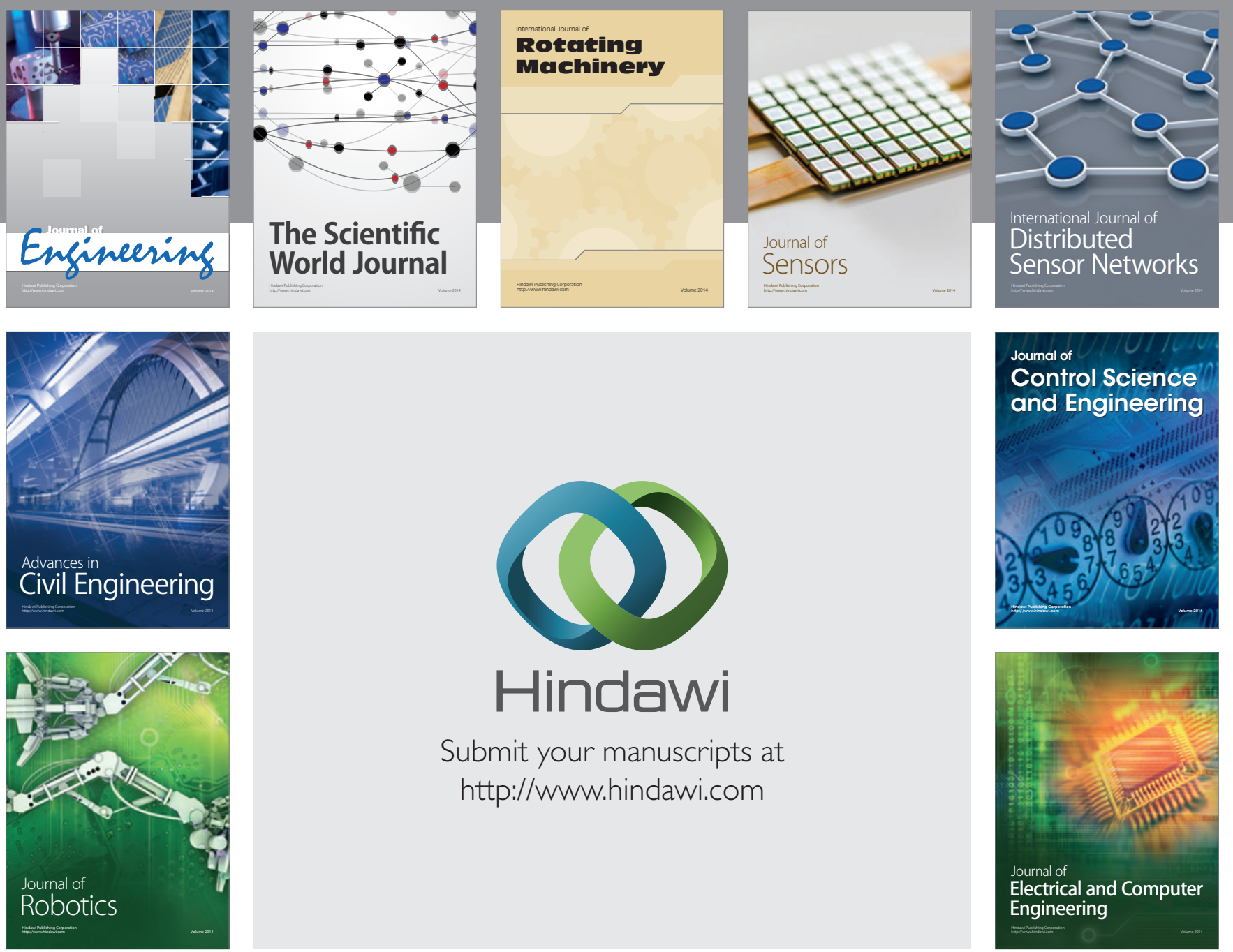

Submit your manuscripts at

http://www.hindawi.com
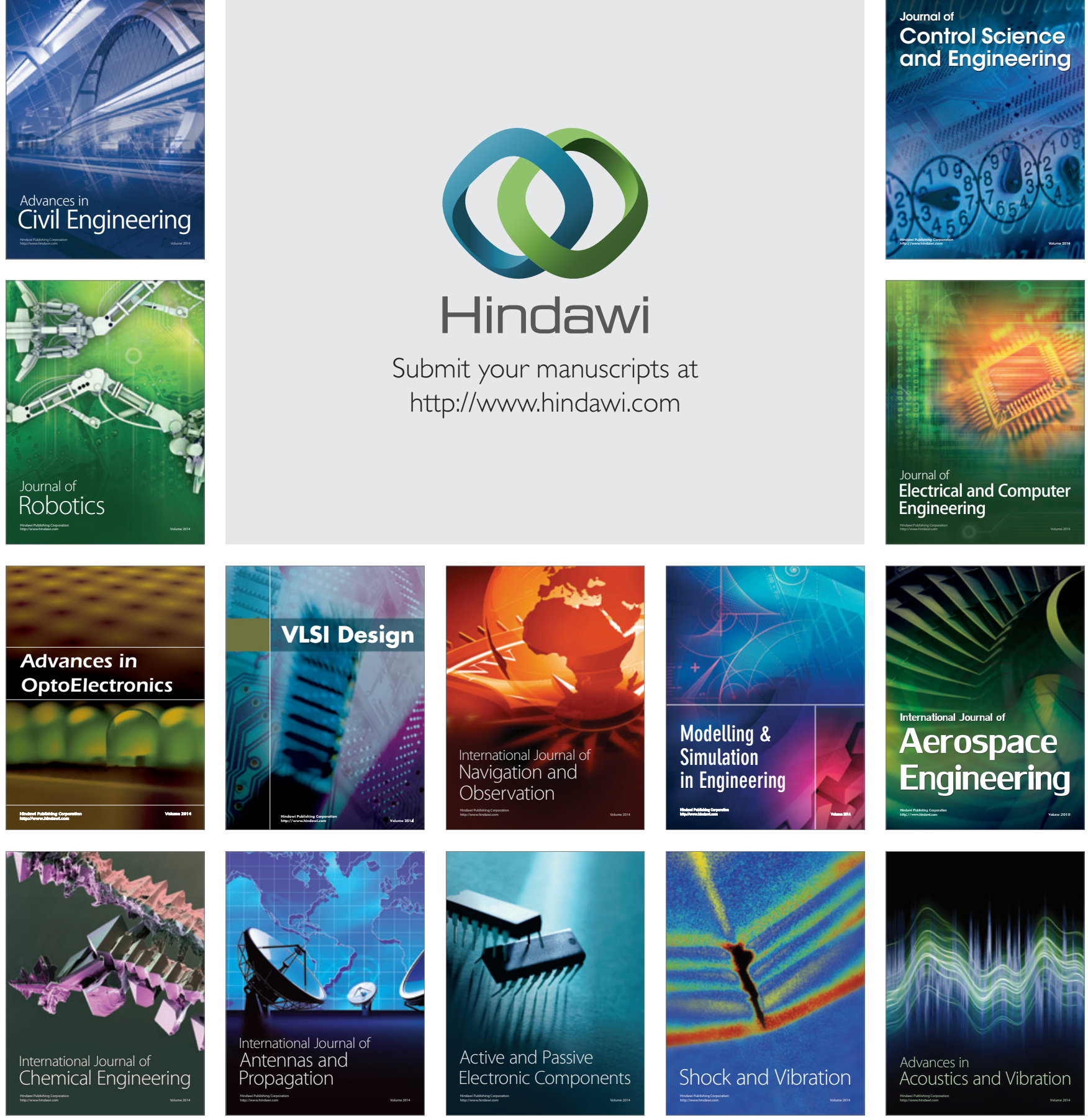